\title{
Does Bose-Einstein condensation of CMB photons cancel $\mu$ distortions created by dissipation of sound waves in the early Universe?
}

\author{
R. Khatri ${ }^{1}$, R. A. Sunyaev ${ }^{1,2,3}$, and J. Chluba ${ }^{4}$ \\ 1 Max Planck Institut für Astrophysik, Karl-Schwarzschild-Str. 1, 85741 Garching, Germany \\ e-mail: khatri@mpa-garching.mpg.de \\ 2 Space Research Institute, Russian Academy of Sciences, Profsoyuznaya 84/32, 117997 Moscow, Russia \\ 3 Institute for Advanced Study, Einstein Drive, Princeton, New Jersey 08540, USA \\ ${ }^{4}$ Canadian Institute for Theoretical Astrophysics, 60 St George Street, Toronto, ON M5S 3H8, Canada
}

Received 3 October 2011 / Accepted 12 February 2012

\begin{abstract}
The difference in the adiabatic indices of photons and non-relativistic baryonic matter in the early Universe causes the electron temperature to be slightly lower than the radiation temperature. Comptonization of photons with colder electrons results in the transfer of energy from photons to electrons and ions, owing to the recoil effect (spontaneous and induced). Thermalization of photons with a colder plasma results in the accumulation of photons in the Rayleigh-Jeans tail, aided by stimulated recoil, while the higher frequency spectrum tries to approach Planck spectrum at the electron temperature $T_{\gamma}^{\text {final }}=T_{\mathrm{e}}<T_{\gamma}^{\text {initial }}$; i.e., Bose-Einstein condensation of photons occurs. We find new solutions of the Kompaneets equation describing this effect. No actual condensate is, in reality, possible since the process is very slow and photons drifting to low frequencies are efficiently absorbed by bremsstrahlung and double Compton processes. The spectral distortions created by Bose-Einstein condensation of photons are within an order of magnitude (for the present range of allowed cosmological parameters), with exactly the same spectrum but opposite in sign, of those created by diffusion damping of the acoustic waves on small scales corresponding to comoving wavenumbers $45<k<10^{4} \mathrm{Mpc}^{-1}$. The initial perturbations on these scales are completely unobservable today due to their being erased completely by Silk damping. There is partial cancellation of these two distortions, leading to suppression of $\mu$ distortions expected in the standard model of cosmology. The net distortion depends on the scalar power index $n_{\mathrm{s}}$ and its running $\mathrm{d} n_{\mathrm{s}} / \mathrm{d} \ln k$, and may vanish for special values of parameters, for example, for a running spectrum with, $n_{\mathrm{s}}=1, \mathrm{~d} n_{\mathrm{s}} / \mathrm{d} \ln k=-0.038$. We arrive at an intriguing conclusion: even a null result, non-detection of $\mu$-type distortion at a sensitivity of $10^{-9}$, gives a quantitative measure of the primordial small-scale power spectrum.
\end{abstract}

Key words. radiation mechanisms: thermal - scattering - cosmic background radiation - cosmological parameters cosmology: theory - early Universe

\section{Introduction}

In the early Universe we have two strongly interacting fluids: cosmic microwave background $(\mathrm{CMB})$ radiation and plasma (=ions + electrons). Electrons and CMB are strongly coupled due to the Compton process with the rate of energy transfer from CMB to electrons given by $R_{\text {compton }} \sim 1.4 \times 10^{-20}(1+z)^{4} \mathrm{~s}^{-1}$. This is much faster than the expansion rate, $H(z) \sim 2.1 \times$ $10^{-20}(1+z)^{2} \mathrm{~s}^{-1}{ }^{1}$, and keeps $T_{\gamma}-T_{\mathrm{e}} \ll T_{\gamma}$ at redshifts $z \gtrsim 200$, where $T_{\gamma}$ is the effective temperature of radiation (to be defined below) and $T_{\mathrm{e}}$ is the electron temperature. Ions and electrons exchange energy via Coulomb collisions at a rate $R_{\text {coulomb }} \sim 3 \times 10^{-9}(1+z)^{3 / 2} \mathrm{~s}^{-1}$ (Lifshitz \& Pitaevskii 1981) keeping $T_{\mathrm{e}}-T_{\mathrm{i}} \ll T_{\mathrm{e}}$, where $T_{\mathrm{i}}$ is the temperature of ions. Thus we have $T_{\gamma} \approx T_{\mathrm{e}} \approx T_{\mathrm{i}}$ to high precision at $z>200$. Non-relativistic matter has an adiabatic index of $5 / 3$, while the adiabatic index of radiation is $4 / 3$. Adiabatic cooling due to the

\footnotetext{
1 We give the formula that is valid during radiation domination just for illustration. The Hubble rate remains much lower than the Comptonization rate at $z>200$ and than the Coulomb rate at even lower redshifts.
}

expansion of the Universe thus makes the matter cool faster than the radiation, while Comptonization tries to maintain matter and radiation at the same temperature by transferring energy from radiation to matter. As a result, the energy of radiation decreases, while the number of photons is conserved (neglecting absorption of photons by bremsstrahlung and double Compton). This effect was discussed by Zeldovich et al. (1968) and Peebles (1968) and leads to a decrease in the temperature of plasma compared to the radiation temperature at $z \lesssim 200$, when Comptonization becomes inefficient because of depletion of electrons from recombination. It also drives the $21-\mathrm{cm}$ line spin temperature to below the radiation temperature, raising the possibility of $21-\mathrm{cm}$ absorption of $\mathrm{CMB}$ photons by neutral hydrogen. This transfer of energy from CMB to matter happens at all redshifts, as long as there is significant free electron number density, and results in deviations in CMB spectrum from the blackbody. Recently, Chluba \& Sunyaev (2012) have numerically studied these deviations from the blackbody in the CMB spectrum. Below we demonstrate that the problem of spectral deviations of CMB due to loss of energy to plasma, in the case of small spectral deviations and small Compton parameter $y$, has a simple analytic solution, when photon production and destruction is neglected. 
The resulting flow of photons towards low frequencies as the spectrum tries to approach the Planck spectrum is in fact the Bose-Einstein condensation of photons. The Bose-Einstein condensation of photons unfortunately does not progress very far in reality, because low-frequency photons are efficiently absorbed by bremsstrahlung and double Compton processes, and the Compton process freezes out as the electron density falls owing to the expansion of the Universe and recombination.

Early papers about energy release in the early Universe were concerned with exotic sources such as annihilation of matter and antimatter, primordial turbulence, decay of new unstable particles, unwinding of topological defects like domain walls, and cosmic strings. Experiments beginning with COBE FIRAS (Fixsen et al. 1996) have been unable to find any significant distortions in the CMB from blackbody. New proposals like PIXIE (Kogut et al. 2011) are demonstrating that a tremendous increase in the sensitivity is possible in the future experiments. PIXIE is proposed to be 1000 times more sensitive than COBE FIRAS. There is hope that this is still not the last word, and even higher sensitivity might become possible in the future. Under these circumstances we decided to check what minimum levels of deviations from blackbody spectrum are expected in the standard cosmological model (Komatsu et al. 2011). In this paper we only consider the spectral distortions arising before the end of the dark ages and beginning of reionization. We demonstrate that, in the absence of decay and annihilation of new unknown particles or any other new physics beyond the standard model, there are only three key reasons for significant global spectral distortions in the $\mathrm{CMB}$.

1. Bose-Einstein condensation of CMB photons due to the difference in the adiabatic indices of non-relativistic plasma and photons.

2. The energy release due to dissipation of sound waves and initial perturbations due to Silk damping (Silk 1968) and the second-order Doppler effect due to non-zero peculiar velocity of electrons and baryons in the CMB rest frame, both leading to the superposition of blackbodies (Zeldovich et al. 1972) in the electron rest frame.

3. The cosmological recombination radiation from hydrogen and helium (Dubrovich 1975; Peebles 1968; Zeldovich et al. 1968).

We omit the distortions caused by the cosmological recombination process (e.g., see Chluba \& Sunyaev 2006; Sunyaev \& Chluba 2009, for more details and references therein) in the discussion below. They have narrow features and different continuum spectrum and can be distinguished from $y$ and $\mu$ distortions from the first two mechanisms. Cosmological recombination radiation can, however, become comparable to or larger than those discussed here (Chluba \& Sunyaev 2012), if the distortions from the first and second processes partially cancel. We also note that for any experiment with finite beam size there will be a mixing of blackbodies in the beam owing to the temperature fluctuations on the last scattering surface on scales smaller than the beam size, leading to inevitable $y$ distortions of magnitude $\sim(\Delta T / T)^{2} \sim 10^{-9}-10^{-10}$ (Chluba \& Sunyaev 2004).

In this paper we find new solutions for the Kompaneets equation describing the CMB spectral distortions arising from process 1 described above. A second mechanism results in a $y$-distortion and heating of the electrons at low redshifts. At high redshifts Comptonization of $\mathrm{CMB}$ on the hotter electrons converts the $y$-type distortion to a $\mu$-type distortion (Sunyaev \& Zeldovich 1970b; Illarionov \& Sunyaev 1975b). We demonstrate below that these two sources of distortions work against each other, individually resulting in spectral distortions with opposite signs but the same spectral shape in any part of the CMB spectrum.

The cooling of electrons and the corresponding spectral distortions are easy to calculate and only depend on the standard cosmological parameters, such as baryon to photon number density $n_{\mathrm{B}} / n_{\gamma}$ and helium fraction, which decide the amount of energy losses by the CMB, the Hubble constant $\left(H_{0}\right)$, and densities of constituents of the Universe, which in turn determine the expansion rate and thus the efficiency of Comptonization. On the other hand, the energy released by dissipation of sound waves crucially depends on the power in small-scale fluctuations, and thus the spectral index $\left(n_{\mathrm{s}}\right)$ in the standard cosmological model, in addition to the other well-measured parameters of the standard cosmological model (Komatsu et al. 2011). It is interesting to note that for a spectrum with constant $n_{\mathrm{S}}$ the energy release due to dissipation exceeds the energy losses to adiabatic cooling of baryons, and there is net heating of electrons. For a primordial spectrum with a running spectral index, the role of Comptonization with colder electrons and Bose-Einstein condensation can become dominant, with the spectral distortions changing sign, and there can be net cooling of electrons and a corresponding decrease of CMB entropy per baryon (specific entropy). Additional increase in entropy during the recombination epoch due to superposition of blackbodies, free streaming as well as Silk damping and the second-order Doppler effect only produce $y$-type distortions and can be distinguished from the $\mu$ distortions created in the earlier epoch.

\section{Thermodynamic equilibrium in the early Universe}

We can obtain the energy losses due to adiabatic cooling of baryons under the assumption that Compton scattering, bremsstrahlung, and double Compton scattering can maintain full thermodynamic equilibrium between the electrons and photons. This is true to high accuracy at $z>10^{6}$ while at lower redshifts, although bremsstrahlung and double Compton scattering cannot help in restoring a Planck spectrum at all frequencies, Comptonization keeps the electron temperature equal to the effective radiation temperature (cf. Eq. (16)) to high precision until $z \sim 200$. In the thermodynamic equilibrium, entropy is conserved, and we can use this to calculate the energy losses due to the expansion of the Universe without referring to Comptonization, since it is not really important which physical process is responsible for the equilibrium.

We start with the thermodynamic relation,

$T_{\gamma} \mathrm{d} S=\mathrm{d} U+P \mathrm{~d} V-M \mathrm{~d} N$

where $S$ is the entropy, $U$ total thermal energy, $P$ pressure, $V$ volume, $M$ chemical potential, and $N$ the number of particles. $T_{\gamma}$ is the common temperature of photons, ions, and electrons. We can ignore the last term for ions and electrons since their number is fixed after big bang nucleosynthesis ends ${ }^{2}$. For photons as well we can ignore the last term if we assume that their chemical potential is 0 , which is true in full thermodynamic equilibrium. With this assumption we can write the equation for total entropy per baryon, $\sigma=s / n_{\mathrm{B}}$, where $s$ is the entropy density and $n_{\mathrm{B}}$ is

\footnotetext{
2 During recombination, the number of particles changes and the departure from thermodynamic equilibrium also becomes significant. The following calculations are therefore strictly valid only before recombination.
} 
R. Khatri et al.: Does Bose-Einstein condensation of CMB photons cancel $\mu$ distortions...

the baryon number density:

$$
\begin{array}{r}
\mathrm{d} \sigma=\frac{\mathrm{d}\left(E / n_{\mathrm{B}}\right)+P \mathrm{~d}\left(1 / n_{\mathrm{B}}\right)}{T_{\gamma}} \\
E=a_{\mathrm{R}} T_{\gamma}^{4}+\frac{3}{2} N n_{\mathrm{B}} k_{\mathrm{B}} T_{\gamma} \\
P=\frac{1}{3} a_{\mathrm{R}} T_{\gamma}^{4}+N n_{\mathrm{B}} k_{\mathrm{B}} T_{\gamma} .
\end{array}
$$

Here $a_{\mathrm{R}}$ is the radiation constant and $N$ the number of nonrelativistic particles per baryon, so that $N n_{\mathrm{B}}=n_{\mathrm{eb}}$. We now integrate Eq. (2) to obtain

$\sigma=\frac{4 a_{\mathrm{R}} T_{\gamma}^{3}}{3 n_{\mathrm{B}}}+N k_{\mathrm{B}} \ln \left(\frac{T_{\gamma}^{3 / 2}}{n_{\mathrm{B}} C}\right)$,

where $C$ is an arbitrary constant of integration, which is not important for our calculation. The first term above is the contribution from photons, and it is clear that in the absence of second term, $T_{\gamma} \propto(1+z)$. The second term is the contribution of nonrelativistic particles and causes the temperature to drop slightly faster in order to conserve the entropy per baryon. We can write $T_{\gamma}=T(1+t)$, where $T=T_{\mathrm{i}}(1+z) /\left(1+z_{i}\right)$ is the background temperature proportional to $1+z$, and $t \ll 1$ is the fractional deviation from this law. We can take the initial deviation $t\left(z_{i}\right)=0$ at initial redshift $z_{i}$ and then calculate the subsequent energy losses to adiabatic cooling of baryons at later redshifts. Thus we can write $\sigma$, correct to leading order in $t$, as

$\sigma=\frac{4 a_{\mathrm{R}} T^{3}}{3 n_{\mathrm{B}}}+\frac{4 a_{\mathrm{R}} T^{3}(3 t)}{3 n_{\mathrm{B}}}+N k_{\mathrm{B}} \ln \left(\frac{T^{3 / 2}}{n_{\mathrm{B}} C}\right)+\frac{3 N k_{\mathrm{B}} t}{2}$.

Equating the above to the initial entropy $\sigma_{i}=\sigma\left(z_{i}\right)$ with $t \equiv$ $0, T \equiv T_{\mathrm{i}}$, we get upon solving for $t$ (and ignoring the last term in Eq. (6) compared to the second term)

$$
\begin{aligned}
t & =\frac{3 N n_{\mathrm{B}} k_{\mathrm{B}}}{8 a_{\mathrm{R}} T^{3}} \ln \left(\frac{1+z}{1+z_{i}}\right) \\
& =\frac{E_{\mathrm{B}}}{4 E_{\gamma}} \ln \left(\frac{1+z}{1+z_{i}}\right) \\
& =-\frac{5.9 \times 10^{-10}}{4} \ln \left(\frac{1+z_{i}}{1+z}\right)
\end{aligned}
$$

with $\Delta E / E=4 t$, since $E_{\gamma} \propto T^{4}$, and $E_{\mathrm{B}}=\frac{3}{2} k_{\mathrm{B}} n_{\mathrm{eb}} T_{\mathrm{e}}$. We note that $t$ is negative since $z_{i}>z$ and there is net energy loss. $\ln \left(z_{\max } / z_{\min }\right) \sim 10$ for the redshift range of interest, $200<$ $z<2 \times 10^{6}$, where a mixture of $\mu$ and $y$-type distortions are produced. This result for the total energy extraction is consistent with the estimate obtained by Chluba (2005) and Chluba \& Sunyaev (2012).

To estimate the magnitude of total distortions, $\mu$ and $y$-type, we take $z_{\max }=z_{i}=2 \times 10^{6}$, since at greater redshifts spectral distortions are rapidly destroyed by bremsstrahlung and double Compton processes and Comptonization. The latter redistributes photons over the whole spectrum. For $z_{\min }=200$ we get $\Delta E / E=5.4 \times 10^{-9}$. This transfer of energy from radiation to baryons due to a difference in their adiabatic indices results in an inevitable distortion and Bose-Einstein condensation of photons. We show below that the distortion has a magnitude of $Y_{\mathrm{BEC}} \sim(1 / 4) \Delta E / E \sim 10^{-9}$. We define parameter $Y_{\mathrm{BEC}}$ and prove this result below. The corresponding deviation of the electron temperature from the equilibrium temperature of electrons in the radiation field is described by Eq. (16) and is $\left(T_{\gamma}-T_{\mathrm{e}}\right) / T_{\gamma} \approx 10^{-12}$ at $z=10^{6}$ growing to $\sim 0.01$ at $z=500$.
In the Universe there are also inevitable processes like the dissipation of acoustic waves and SZ effect from reionization, which would lead to a normal SZ effect of similar or greater magnitude. Due to the additivity of small spectral distortions, these effects will cancel the $Y_{\mathrm{BEC}}$ distortions, and this diminishes the hope that this Bose-Einstein condensation will ever be observed. Nevertheless it is important to stress that in the standard cosmology there was an epoch $10^{6}>z>1000$ when BoseEinstein condensation of CMB photons due to the difference in the adiabatic indices of matter and radiation was able to create a peculiar deviation of CMB spectrum from the blackbody.

\section{Alternative direct calculation of energy losses in $\mathrm{CMB}$}

We would like to remind the reader that adiabatic cooling of baryons leads to the cooling of radiation, but Comptonization itself conserves the number of photons. We can write the equation for the evolution of average thermal energy density $E_{\mathrm{B}}$ in baryons before recombination as

$\frac{\mathrm{d} E_{\mathrm{B}}}{\mathrm{d} z} \equiv \frac{3}{2} k_{\mathrm{B}} T_{\mathrm{e}} \frac{\mathrm{d} n_{\mathrm{eb}}}{\mathrm{d} z}+\frac{3}{2} k_{\mathrm{B}} n_{\mathrm{eb}} \frac{\mathrm{d} T_{\mathrm{e}}}{\mathrm{d} z}$.

The derivative in the first term on the right-hand side, $\mathrm{d} n_{\mathrm{eb}} / \mathrm{d} z=$ $3 n_{\mathrm{eb}} /(1+z)$, is just the decrease in number density of particles (ions and electrons) with redshift $z$ due to the Hubble expansion, with $n_{\mathrm{eb}}=\rho_{\mathrm{b}} / \mu_{\mathrm{mol}}$, where $\rho_{\mathrm{b}}$ is the baryon mass density and $\mu_{\mathrm{mol}}$ the mean molecular weight. The change in temperature has contributions from adiabatic cooling and also from energy gained from CMB by Comptonization:

$\frac{\mathrm{d} T_{\mathrm{e}}}{\mathrm{d} z}=\frac{2 T_{\mathrm{e}}}{1+z}-\frac{2}{3 k_{\mathrm{B}} n_{\mathrm{eb}}} S_{\text {Compton }}$

where $S_{\text {Compton }}=4 k_{\mathrm{B}} E_{\gamma} n_{\mathrm{e}} \sigma_{\mathrm{T}}\left(T_{\gamma}-T_{\mathrm{e}}\right) / m_{\mathrm{e}} c H(z)(1+z)$ is the energy transfer rate per unit volume from radiation to baryons by Compton scattering, $n_{\mathrm{e}}$ is the number density of free electrons, $\sigma_{\mathrm{T}}$ the Thomson scattering cross section, $k_{\mathrm{B}}$ the Boltzmann constant, $E_{\gamma}$ the energy density of radiation, $c$ the speed of light, $m_{\mathrm{e}}$ the mass of electron, and $H(z)$ the Hubble parameter. That redshift decreases with increasing time, so the terms with "+" sign are cooling terms and terms with "-" sign are heating terms. The change in photon energy density can therefore be written as

$$
\begin{aligned}
\frac{\mathrm{d} E_{\gamma}}{\mathrm{d} z} & =\left(\frac{\mathrm{d} E_{\gamma}}{\mathrm{d} z}\right)^{\text {adiabatic }}+\left(\frac{\mathrm{d} E_{\gamma}}{\mathrm{d} z}\right)^{\text {Compton }} \\
& =\frac{4 E_{\gamma}}{1+z}+S_{\text {Compton }}
\end{aligned}
$$

where the first term is just the adiabatic cooling due to the expansion of the Universe. We can estimate the energy transfer, $S_{\text {Compton }}$, by noting that the baryon temperature $T_{\mathrm{e}}=T_{\gamma} \propto 1+z$ to a high accuracy until $z \sim 200$, where $T_{\gamma}=2.725(1+z) \mathrm{K}$ is the CMB temperature. Using this to evaluate the total derivative of electron temperature $\mathrm{d} T_{\mathrm{e}} / \mathrm{d} z$ on the left-hand side of Eq. (9) and the first term on the right-hand side, we get

$$
\begin{aligned}
S_{\text {Compton }} & =\frac{3}{2} k_{\mathrm{B}} n_{\mathrm{eb}} \frac{2 T_{\gamma}}{1+z}-\frac{3}{2} k_{\mathrm{B}} n_{\mathrm{eb}} \frac{\mathrm{d} T_{\gamma}}{\mathrm{d} z} \\
& =\frac{E_{\mathrm{B}}}{1+z} .
\end{aligned}
$$


Thus we have fractional rate at which energy is lost by radiation to baryons

$$
\frac{\left(\mathrm{d} E_{\gamma} / \mathrm{d} z\right)^{\text {Compton }}}{E_{\gamma}}=\frac{S_{\text {Compton }}}{E_{\gamma}}=\frac{E_{\mathrm{B}} / E_{\gamma}}{(1+z)}=\frac{5.9 \times 10^{-10}}{1+z} .
$$

We can now calculate the total fractional energy losses of radiation that contribute to the spectral distortions between redshifts $z_{\min }$ and $z_{\max }$,

$$
\begin{aligned}
\frac{\Delta E}{E} & =\int_{z_{\max }}^{z_{\min }} \frac{\mathrm{d} E_{\gamma}}{E_{\gamma}} \\
& =\int_{z_{\max }}^{z_{\min }} \frac{\mathrm{d} z}{E_{\gamma}}\left(\frac{\mathrm{d} E_{\gamma}}{\mathrm{d} z}\right)^{\text {Compton }} \\
& =-5.9 \times 10^{-10} \ln \left(\frac{1+z_{\max }}{1+z_{\min }}\right) .
\end{aligned}
$$

We have integrated $\mathrm{d} E / E$, since immediate distortions are proportional to $\Delta E / E$, and the distortions can be added linearly if they are small. This is the same result as Eq. (7).

\section{Kompaneets equation}

The interaction of radiation with electrons through Compton scattering or Comptonization is described by the Kompaneets equation (Kompaneets 1956) in the Fokker-Planck approximation, when the energy transfer in each scattering is small compared to temperature, and the incoming photon distribution is wide compared to the width of the scattering kernel:

$\frac{\partial n}{\partial y}=\frac{1}{x^{2}} \frac{\partial}{\partial x} x^{4}\left(n+n^{2}+\frac{T_{\mathrm{e}}}{T_{\mathrm{BB}}} \frac{\partial n}{\partial x}\right)$,

where we have defined the Compton parameter

$y\left(z, z_{\max }\right)=-\int_{z_{\max }}^{z} \mathrm{~d} z \frac{k_{\mathrm{B}} \sigma_{\mathrm{T}}}{m_{\mathrm{e}} c} \frac{n_{\mathrm{e}} T_{\mathrm{BB}}}{H(1+z)}$,

which is convenient to use instead of time or redshift. We start our calculation at the reference redshift $z_{\max }$. One can furthermore introduce the Compton equilibrium electron temperature in a radiation field (Levich \& Sunyaev 1971; Zel'Dovich \& Levich 1970)

$\frac{T_{\mathrm{e}}^{\mathrm{eq}}}{T_{\mathrm{BB}}}=\frac{\int n(1+n) x^{4} \mathrm{~d} x}{4 \int n x^{3} \mathrm{~d} x}$.

Here $T_{\mathrm{BB}}$ is a reference temperature which is equal to the radiation blackbody temperature if the initial radiation field is a Planck spectrum, and $x=h v / k_{\mathrm{B}} T_{\mathrm{BB}}$ is the dimensionless frequency with $v$ the frequency of photons. The equations written in this way factor out the expansion of the Universe and are applicable to cosmology, as well as non-expanding astrophysical systems. This equation and the Kompaneets equation form a coupled system to be solved simultaneously.

The three terms in the inner brackets of Eq. (14) describe recoil $(n)$, induced recoil $\left(n^{2}\right)$, and the Doppler effect of the thermal motion of electrons $\left(T_{\mathrm{e}} / T_{\mathrm{BB}} \partial n / \partial x\right)$. For $y \ll 1$ and an initial blackbody spectrum with temperature $T_{\mathrm{BB}}, n_{\mathrm{BB}}=1 /\left(\mathrm{e}^{x}-1\right)$, a simple first-order correction to the blackbody spectrum can be found (Zeldovich \& Sunyaev 1969). By approximating ${ }^{3} n+n^{2} \approx$ $-\partial n / \partial x$ in Eq. (14) we get

$\frac{\partial n(x, y)}{\Delta \partial y}=\frac{1}{x^{2}} \frac{\partial}{\partial x}\left[x^{4} \frac{\partial n(x, y)}{\partial x}\right]$

where $\Delta \equiv T_{\mathrm{e}} / T_{\mathrm{BB}}-1$. This particular form of equation emphasizes that the Doppler term dominates over recoil and gives rise to the spectral distortion by boosting low-energy photons to higher frequency. The above equation can be reduced to the diffusion or heat equation (Zeldovich \& Sunyaev 1969) by changing variables, for which the Green's function is well known.

For small distortions, the solution takes a particularly simple form and can be found by substituting $n_{\mathrm{BB}}$ on the right-hand side of Eqs. (14) or (17):

$\Delta n \equiv n\left(x, Y_{\mathrm{SZ}}\right)-n_{\mathrm{BB}}(x)=Y_{\mathrm{SZ}} \frac{x \mathrm{e}^{x}}{\left(\mathrm{e}^{x}-1\right)^{2}}\left[x \frac{\mathrm{e}^{x}+1}{\mathrm{e}^{x}-1}-4\right]$,

where we have defined $Y_{\mathrm{SZ}}=\int_{0}^{y} \Delta \mathrm{d} y$. The SZ parameter is related to fractional energy release by the formula $Y_{\mathrm{SZ}}=$ $(1 / 4) \Delta E_{\gamma} / E_{\gamma}$. This can be obtained by integrating the above spectrum over all frequencies.

\section{New solution of Kompaneets equation for Comptonization of photons with colder plasma}

The Kompaneets equation describes, to lowest order, the competition between the Doppler boosting of low-energy photons to high energies and the down scatter of high-energy photons to low energies by recoil and stimulated recoil. The initial radiation spectrum is blackbody with temperature $T_{\mathrm{BB}}$. If the electrons are also at the same temperature, $T_{\mathrm{e}}=T_{\mathrm{BB}}$, then these two effects cancel each other out exactly. If $T_{\mathrm{e}}>T_{\mathrm{BB}}$, the Doppler boosting becomes stronger than recoil and we have the normal SZ effect in the limit of small $y$ parameter. If $T_{\mathrm{e}}<T_{\mathrm{BB}}$ then Doppler boosting is weaker and we have a net movement of photons from high to low energies, which is the complete opposite of the SZ effect. But since at $T_{\mathrm{e}}=T_{\mathrm{BB}}$ the two effects exactly balance each other out, at linear order the spectral distortions for $T_{\mathrm{e}}<T_{\mathrm{BB}}$ would be exactly the same as for $T_{\mathrm{e}}>T_{\mathrm{BB}}$, just with the opposite sign.

For $T_{\mathrm{e}}<T_{\mathrm{BB}}$, we can write the approximate Kompaneets equation by approximating $\partial n / \partial x \approx-\left(n+n^{2}\right)$ in Eq. (14),

$\frac{\partial n}{\partial y}=\left(1-\frac{T_{\mathrm{e}}}{T_{\mathrm{BB}}}\right) \frac{1}{x^{2}} \frac{\partial}{\partial x} x^{4}\left(n+n^{2}\right)$.

Analytic solutions can be obtained by the standard method of characteristics for the recoil $(n)$ and induced recoil $\left(n^{2}\right)$ terms individually, and they consist of photons moving down the frequency axes at a speed proportional to $x^{2}$ for the recoil term (Arons 1971; Illarionov \& Syunyaev 1972) and at a speed proportional to the $x^{2} n$ for the induced recoil term (Zel'Dovich \& Levich 1969; Syunyaev 1971). To get the linear solution, we can just substitute $n(x)=n_{\mathrm{BB}}(x)$ on the righthand side of Eq. (19) and define the parameter for the amplitude of distortion in the case $T_{\mathrm{e}}<T_{\mathrm{BB}}$ as $Y_{\mathrm{BEC}}=\int_{0}^{y}\left(1-T_{\mathrm{e}} / T_{\mathrm{BB}}\right) \mathrm{d} y$, the result is Eq. (18) with $Y_{\mathrm{SZ}}=-Y_{\mathrm{BEC}}$. We should emphasize that the linear solution, Eq. (18), can be arrived at in a straightforward way by just substituting $n(x)=n_{\mathrm{BB}}(x)$ on the righthand side of

${ }^{3}$ For a Bose-Einstein distribution with constant chemical potential this expression is an identity. 
R. Khatri et al.: Does Bose-Einstein condensation of CMB photons cancel $\mu$ distortions...

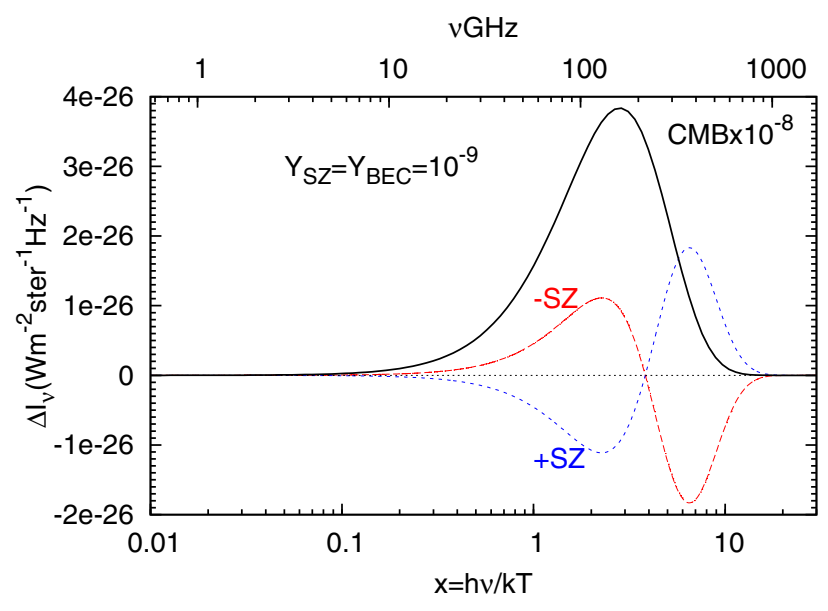

Fig. 1. Difference in intensity from the blackbody radiation for normal SZ effect with $Y_{\mathrm{SZ}}=10^{-9}$ and negative SZ effect with $Y_{\mathrm{BEC}}=10^{-9}$.

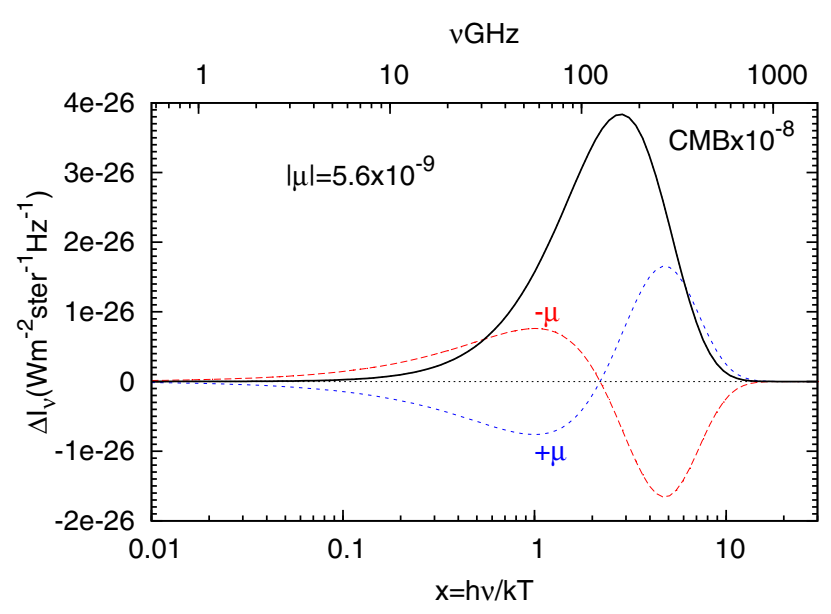

Fig. 2. Difference in intensity from the blackbody radiation for $\mu$ distortion defined by the equation with $n(v)=1 /\left(\mathrm{e}^{h v / k_{\mathrm{B}} T_{\mu}+\mu}-1\right) . T_{\mu}$ is the temperature to which the spectrum relaxes for an initial energy addition/loss of $\Delta E / E$ with $\Delta T_{\mu} / T=0.64 \Delta E / E= \pm 2.5 \times 10^{-9}$. $\Delta E / E= \pm 4 \times 10^{-9}$ is the energy addition/loss that gives rise to SZ distortion of $10^{-9}$. The dimensionless chemical potential $\mu$ is given by $\mu=2.2 \Delta T / T= \pm 5.6 \times 10^{-9}$.

the full Kompaneets equation, Eq. (14), without any restriction on $T_{\mathrm{e}}$. However the dominant physical effects when $T_{\mathrm{e}}>T_{\mathrm{BB}}$ (Doppler) and when $T_{\mathrm{e}}<T_{\mathrm{BB}}$ (recoil and induced recoil) are completely different and that in equilibrium they balance each other out exactly gives us the same mathematical form of the linear solution in both cases.

In the Rayleigh-Jeans part of the spectrum we now have an increase in the brightness temperature of $\Delta T / T=+2 Y_{\mathrm{BEC}}$, which is independent of frequency and thus maintains the Rayleigh-Jeans shape of the spectrum. In the Wien part we have a decrease in intensity $\Delta I / I=\Delta n / n \approx-x^{2} Y_{\mathrm{BEC}}$. Figure 1 shows the difference in intensity from the blackbody radiation for normal SZ effect with $Y_{\mathrm{SZ}}=10^{-9}$ and the negative SZ effect with $Y_{\mathrm{BEC}}=10^{-9}$. Figure 2 shows the spectrum that would be achieved at a high value of the Compton parameter $y$. It is a Bose-Einstein spectrum with the dimensionless chemical potential $^{4} \mu$ defined by the equation with $n(v)=1 /\left(\mathrm{e}^{h v / k_{\mathrm{B}} T_{\mu}+\mu}-1\right)$,

\footnotetext{
4 This definition has a sign difference with respect to the usual definition of the chemical potential in thermodynamics (Landau \& Lifshitz 1980) used in Eq. (1).
}

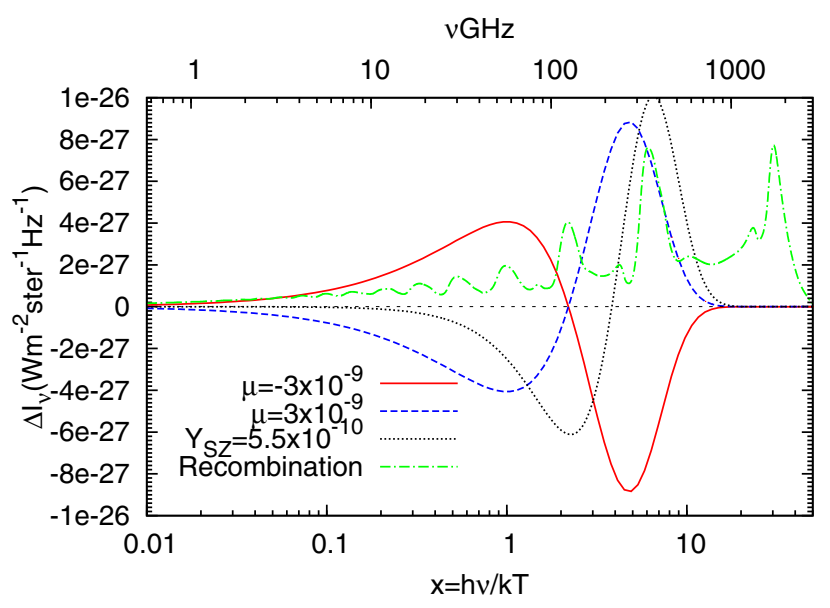

Fig. 3. Comparison of positive and negative $\mu$-type distortions expected in the early Universe (to be calculated in later sections) with the $y$-type distortions before reionization and the cosmological recombination spectrum (taken from Chluba \& Sunyaev 2006; Rubiño-Martín et al. 2008) for illustration.

where $T_{\mu}$ is the temperature to which the spectrum relaxes for an initial energy addition/loss of $\Delta E / E$ with $\Delta T_{\mu} / T=$ $0.64 \Delta E / E= \pm 2.5 \times 10^{-9}$ (Illarionov \& Sunyaev 1975a), and $\Delta E / E= \pm 4 \times 10^{-9}$ is the energy addition/loss that gives rise to SZ distortion of $10^{-9}$. The chemical potential $\mu$ is given by ${ }^{5}$ $\mu=2.2 \Delta T / T= \pm 5.6 \times 10^{-9}$. This is the spectrum that an initial spectrum with $Y_{\mathrm{BEC}}, Y_{\mathrm{SZ}}=10^{-9}$ will approach at high $y$ at $x \gg \mu \sim 10^{-9}$. The frequency at which the distortion crosses zero is at $x=2.19$ compared to $x=3.83$ for the SZ distortion in Fig. 1.

The $y$-type and $\mu$-type distortions expected in the early Universe (calculated in the later sections) are compared with the cosmological recombination spectrum (Rubiño-Martín et al. 2006) in Fig. 3. Clearly $\mu$ type distortions have a different spectral shape than the recombination radiation (both from hydrogen (Chluba \& Sunyaev 2006) and helium (Rubiño-Martín et al. 2008)) and $y$-type distortions and can be distinguished from the last two. This is very important because the information in the $\mu$ type distortions about the early Universe physics can be extracted. On the other hand, the $y$-type distortions from the early Universe get swamped by the much larger $y$-type distortions from the low redshifts and the two contributions are difficult to separate. The $\mu$ distortions expected from the early Universe also have higher magnitude than the recombination spectrum in the Rayleigh-Jeans part of the spectrum but has no quasi-periodic structure like the cosmological recombination radiation.

This flow of photons toward lower frequencies as the spectrum tries to approach the Planck spectrum due to recoil, and induced recoil is Bose-Einstein condensation of photons (Illarionov \& Sunyaev 1975a; Landau \& Lifshitz 1980). We show the evolution of the spectrum (solution of the coupled system of Eqs. (14) and (16)) starting with the initial distortion given by Eq. (18) with $-Y_{\mathrm{SZ}}=Y_{\mathrm{BEC}}=10^{-5}$ in Fig. 4. In the Rayleigh-Jeans region intensity $I_{v} \propto T_{\text {eff }}$ and therefore $\Delta T / T=\Delta I / I$. The initial evolution is similar to that of a spectrum with positive $Y_{\mathrm{SZ}}$ with the photon distribution approaching a Bose-Einstein spectrum defined by $n(v)=1 /\left(\mathrm{e}^{h v / k_{\mathrm{B}} T_{\mathrm{e}}+\mu}-1\right)$ with negative $\mu$ (marked "-ve BE" in the figure). We should

\footnotetext{
5 These relationships can be easily derived using photon number and energy conservation and requiring that the final spectrum have the equilibrium Bose-Einstein distribution.
} 


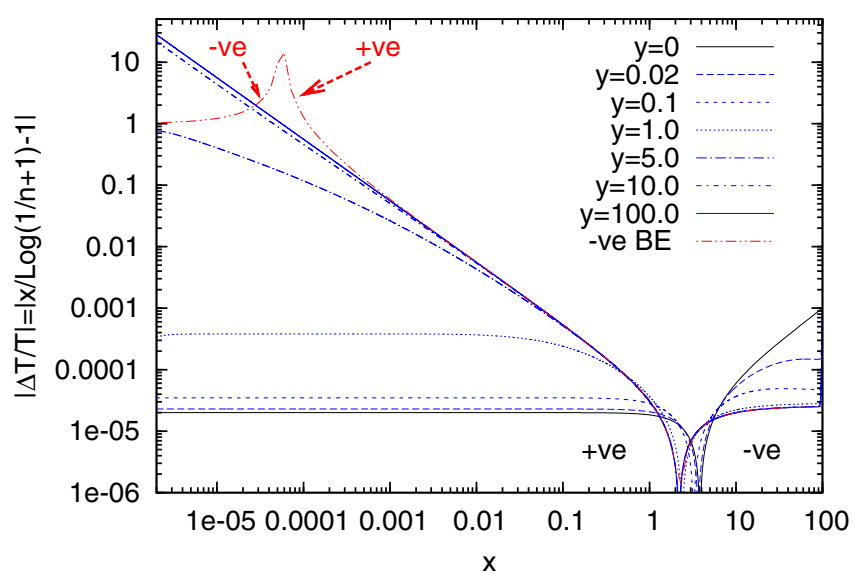

Fig. 4. Evolution of initial spectrum at $y=0$ given by Eq. (18) with $-Y_{\mathrm{SZ}}=Y_{\mathrm{BEC}}=10^{-5} \cdot|\Delta T / T| \equiv\left|T_{\mathrm{eff}} / T-1\right|$ is plotted, where $T_{\mathrm{eff}}$ is the temperature of a blackbody spectrum with the corresponding intensity at a particular frequency. Bose-Einstein spectrum defined by $n(v)=1 /\left(\mathrm{e}^{h v / k_{\mathrm{B}} T_{\mathrm{e}}+\mu}-1\right)$ with negative $\mu$ is labeled "-ve BE", red dashed, double-dotted line in the figure. The distortions are positive in the Rayleigh-Jeans region (labeled "+ve") on the left side and negative in the Wien region (labeled "-ve") on the right side of the figure.

emphasize that there is no singularity in the actual solutions of the Kompaneets equation plotted above. The singularity is just in the mathematical formula, which correctly describes the spectrum at high frequencies, $x \gg|\mu|$. The actual spectrum deviates from the Bose-Einstein spectrum near the singularity (positive everywhere in the Rayleigh-Jeans region) and can be described by a chemical potential decreasing in magnitude with decreasing frequency. The evolution at $y>1$ is therefore very different from the positive $Y_{\mathrm{SZ}}$ case. For $x \gg|\mu|$ the spectrum is the BoseEinstein spectrum at the electron temperature. For $x \ll|\mu|$ there is an excess of photons compared to the Bose-Einstein spectrum which grows with time, a feature of Bose-Einstein condensation. The fractional change in temperature $\Delta T / T$ takes a very simple form in the Rayleigh-Jeans region $(x \ll 1)$ for a Bose-Einstein spectrum and can be understood as follows. It is given, in terms of frequency referred to the electron temperature, $x_{\mathrm{e}} \equiv h v / k T_{\mathrm{e}}$, by

$\frac{\Delta T}{T}=\frac{x_{\mathrm{e}}}{x_{\mathrm{e}}+\mu}-1=\frac{-\mu}{x_{\mathrm{e}}+\mu}$

For $|\mu| \ll x_{\mathrm{e}} \ll 1$ we have $\Delta T / T=-\mu / x_{\mathrm{e}}$. Thus the fractional temperature deviation is positive for negative $\mu$ and negative for the positive $\mu$. For $x_{\mathrm{e}}$ greater than and close to $|\mu|, \mu<0$ the spectral distortions can become very large and exceed unity. Since the high-frequency spectrum is in equilibrium at the electron temperature by $y=10$, the subsequent evolution is very slow, and $|\mu|$ decreases while the electron temperature increases slowly as the photon distribution approaches a Planck spectrum with the extra photons accumulating at low frequencies; i.e., Bose-Einstein condensation happens. The low-frequency spectrum approaches the stationary solution of Kompaneets equation with only the induced recoil term, $n(x) \propto 1 / x^{2}$, with a continuous flow of photons towards $x=0$ (Syunyaev 1971). Then, $T_{\mathrm{e}} / T-1$ just increases from $-2.558 \times 10^{-5}$ to $-2.555 \times 10^{-5}$ in going from $y=10$ to $y=100$ for the chosen energy losses of $\Delta E / E=4 \times 10^{-5}$. The effect discussed below in the real Universe is of magnitude $\sim 10^{-9}$.

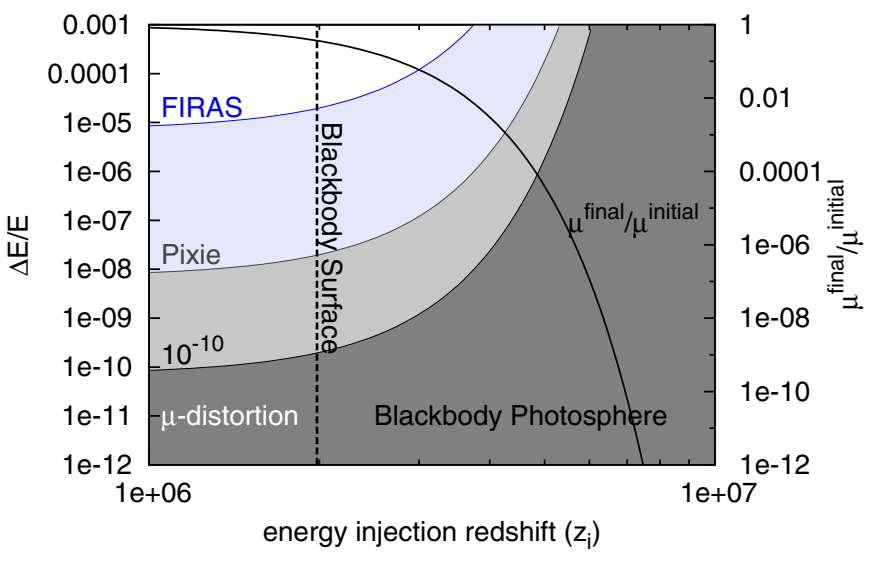

Fig. 5. Regions of the blackbody photosphere probed by different experiments, COBE FIRAS with sensitivity $\mu=10^{-5}$, Pixie with sensitivity $\mu=10^{-8}$ and a hypothetical experiment with sensitivity of $\mu=10^{-10}$. Shaded regions below the curves are allowed and inaccessible to these experiments. We also plot the ratio of final $\mu$ distortion today to the initial $\mu$ distortion as a function of energy injection redshift. We define the blackbody surface as the energy injection redshift such that $\mu^{\text {final }} / \mu^{\text {initial }}=1 / e, z_{\mathrm{bb}}=1.98 \times 10^{6}$.

\section{Bose-Einstein condensation of CMB photons in the early Universe}

In the early Universe, before $z \sim 2 \times 10^{6}$ double Compton and Comptonization destroy any spectral distortions and maintain the Planck spectrum of CMB. For small distortions due to single, quasi-instantaneous episode of energy release, we can write the ratio of final-to-initial $\mu$ as an exponential function of redshift defined by the square root of the product of Comptonization and absorption rates (Sunyaev \& Zeldovich 1970b). For a double Compton process, this formula gives (Danese \& de Zotti 1982)

$G(z)=\frac{\mu^{\mathrm{final}}}{\mu^{\text {initial }}} \approx \mathrm{e}^{-\left(z_{\mathrm{i}} / z_{\mathrm{dc}}\right)^{5 / 2}}$,

where $z_{\mathrm{dc}} \approx 1.98 \times 10^{6}$ defines the "surface of the blackbody photosphere". We call this function $G(z)$ the blackbody visibility function for spectral distortions. We call the region $z>z_{\mathrm{dc}}$ where the initial $\mu$ distortion can be reduced by a factor of more than $e$ the "blackbody photosphere". Thus inside the blackbody photosphere, a Planckian spectrum can be established efficiently. Figure 5 shows how the $\mu$ distortion at high redshifts decreases due to the double Compton and Compton scattering. The regions of the blackbody photosphere allowed by different experiments are shaded.

At redshifts $10^{5}<z<2 \times 10^{6}$, we have the Compton parameter $y>1$, and the Bose-Einstein spectrum with a negative chemical potential is established at $x \gtrsim 0.01^{6}$. At lower frequencies, bremsstrahlung and double Compton create a Planck spectrum corresponding to the electron temperature. At $z<10^{4}$ the Compton parameter $y<0.01$ and the distortions created can be described by the linear solution of Eq. (18). Thus the net distortions created in the early Universe owing to adiabatic cooling of baryons are a linear superposition of distortions corresponding to

${ }^{6}$ Strictly speaking, a Bose-Einstein spectrum is established at $y \sim$ few as can be seen from Fig. 4 However at $y \sim 0.25-1$ the spectrum is already very close to Bose-Einstein at high frequencies. We use this to divide the energy release into $\mu$-type and $y$-type estimates. Exact results are presented elsewhere (Chluba et al. 2012). 


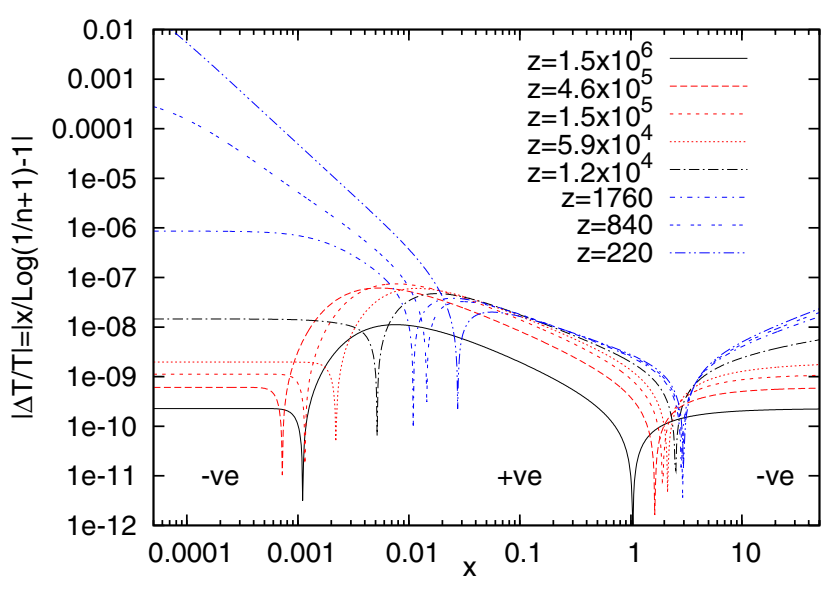

Fig. 6. Evolution of spectral distortions in $\mathrm{CMB}$ in $\Lambda \mathrm{CDM}$ cosmology. The values of total Compton parameter $y=y(100, z)$ for different curves are $\left(z=1.5 \times 10^{6}, y=109\right),\left(z=4.6 \times 10^{5}, y=10\right)$, $\left(z=1.5 \times 10^{5}, y=1\right),\left(z=5.9 \times 10^{4}, y=0.16\right),\left(z=1.2 \times 10^{4}, y=\right.$ $0.005),\left(z=1760, y=3 \times 10^{-5}\right),\left(z=840, y=1.8 \times 10^{-8}\right)$ and $\left(z=220, y=1.2 \times 10^{-10}\right)$. Reference spectrum is the blackbody CMB spectrum at $z=10^{7}$. At low frequencies the spectrum is dominated by double Compton and bremsstrahlung which maintain Planck spectrum at the electron temperature. The zero point at low frequencies moves to the right at low redshifts because bremsstrahlung dominates over the Compton scattering at higher and higher frequencies and absorbs photons, although both are slower than the expansion rate.

different values of $y$, and a few of them are shown in Fig. 6. We show the evolution of actual spectrum (Eqs. (14) and (16)) including the effects of bremsstrahlung and double Compton processes (Burigana et al. 1991; Chluba \& Sunyaev 2012; Hu \& Silk 1993; Illarionov \& Sunyaev 1975a,b) in the $\Lambda$ CDM cosmology with WMAP parameters (Komatsu et al. 2011). The reference spectrum is the blackbody CMB spectrum at $z=10^{7}$. At $x<0.01$ the spectrum is dominated by bremsstrahlung and double Compton processes that destroy the condensing lowfrequency photons. The effect of bremsstrahlung compared to Comptonization becomes stronger with decreasing redshift and can be felt at successively higher frequencies.

At $z \gtrsim 5 \times 10^{4}$ the spectral distortions in the $x \gtrsim 0.01$ region can relax to a Bose-Einstein spectrum due to high value of parameter $y$. Contributions from lower redshifts are described by the analytic solution of Eq. (18). The distortions created at $z<10^{4}$ are just the negative of the usual SZ effect and would be completely overwhelmed by the $\mathrm{SZ}$ effect from reionization at $z \sim 10$ which is expected to be $Y_{\mathrm{SZ}}=\left(k_{\mathrm{B}} T_{\mathrm{e}} / m_{\mathrm{e}} c^{2}\right) \tau_{\mathrm{ri}} \sim$ $10^{-6} \times 0.1=10^{-7}$, where $\tau_{\mathrm{ri}} \sim 0.1$ is the optical depth to the last scattering surface due to reionization and $T_{\mathrm{e}} \sim 10^{4} \mathrm{~K}$ is the average electron temperature during reionization. The distortions created by energy loss at $z>10^{5}$ can, however, be destroyed only by energy injection at $z>10^{5}$ since at lower redshift Comptonization cannot create $\mu$-type distortions.

\section{Energy release from dissipation of acoustic waves during radiation domination}

In the standard cosmology the main source of energy injection at high redshifts is the dissipation of acoustic waves in the baryon-photon plasma (Sunyaev \& Zeldovich 1970b) due to Silk damping (Silk 1968). Sunyaev \& Zeldovich (1970a) first proposed using the resulting spectral distortions to measure the spectral index and power in the small-scale fluctuations, which themselves do not survive. Later during recombination the second-order Doppler effect due to non-zero electron/baryon velocity in the CMB rest frame also becomes important, but at that time only $y$-type distortions similar to the SZ effect arise, and $\mu$ distortions cannot be created. We can estimate the amount of $\mu$ distortions created by dissipation of acoustic waves following Hu et al. (1994) by calculating how the total power in the density fluctuations changes with time because of photon diffusion. The comoving energy density in acoustic waves in the photonbaryon plasma (neglecting the baryon energy density) is given by $Q=\rho_{\gamma} c_{\mathrm{s}}^{2}\left\langle\delta_{\gamma}(\boldsymbol{x})^{2}\right\rangle$, where $\rho_{\gamma}$ is the comoving photon energy density, $c_{\mathrm{s}}^{2} \sim 1 / 3$ is sound speed squared, $\delta_{\gamma}$ is the photon density perturbation at position $\boldsymbol{x}$, and angular brackets denote the ensemble average:

$\left\langle\delta_{\gamma}(\boldsymbol{x})^{2}\right\rangle=\int \frac{\mathrm{d}^{3} k}{(2 \pi)^{3}} P_{\gamma}(k)$,

where $P_{\gamma}(k)=\Delta_{\gamma}^{2}(k) P_{\gamma}^{\mathrm{i}}(k)$, and $P_{\gamma}^{\mathrm{i}}(k)$ is the initial power spectrum. It was shown by Mukhanov \& Chibisov (1981) that primordial fluctuations from inflation can have a spectrum deviating from the scale-invariant Harrison-Zeldovich spectrum, with spectral index $n_{\mathrm{s}}<1$. In the cyclic ekpyrotic models $n_{\mathrm{s}}>1$ is also possible (Lehners et al. 2007). WMAP gives the constraint on curvature perturbation in comoving gauge, $\zeta$. This is related to the gravitational perturbation $\psi$ in the radiation era (assuming neutrinos are free streaming) by the relation $\psi=\zeta /\left(2 / 5 R_{v}+1.5\right)$, where $R_{v}=\rho_{v} /\left(\rho_{\gamma}+\rho_{v}\right) \approx 0.4, \rho_{v}$ is the neutrino energy density and $\delta_{\gamma}^{\mathrm{i}}=-2 \psi\left(\right.$ Ma \& Bertschinger 1995). Thus $P_{\gamma}^{\mathrm{i}}=4 /\left(2 / 5 R_{v}+\right.$ $1.5)^{2} P_{\zeta}=1.45 P_{\zeta}$ and $P_{\zeta}=\left(A_{\zeta} 2 \pi^{2} / k^{3}\right)\left(k / k_{0}\right)^{n_{\mathrm{s}}-1+\frac{1}{2} r\left(\ln k / k_{0}\right)}$, $k_{0}=0.002 \mathrm{Mpc}^{-1}, A_{\zeta}=2.4 \times 10^{-9}$ (Keisler et al. 2011; Komatsu et al. 2011), $r=\mathrm{d} n_{\mathrm{s}} / \mathrm{d} \ln k$ is the running of the index. The transfer function for modes well inside the horizon before recombination is given by

$\Delta_{\gamma} \approx 3 \cos \left(k r_{\mathrm{s}}\right) \mathrm{e}^{-k^{2} / k_{\mathrm{D}}^{2}}$

and the diffusion scale by (Kaiser 1983; Weinberg 2008)

$$
\begin{aligned}
\frac{1}{k_{\mathrm{D}}^{2}} & =\int_{z}^{\infty} \mathrm{d} z \frac{c(1+z)}{6 H(1+R) n_{\mathrm{e}} \sigma_{\mathrm{T}}}\left(\frac{R^{2}}{1+R}+\frac{16}{15}\right) \\
\frac{\mathrm{d}\left(1 / k_{\mathrm{D}}^{2}\right)}{\mathrm{d} z} & =-\frac{c(1+z)}{6 H(1+R) n_{\mathrm{e}} \sigma_{\mathrm{T}}}\left(\frac{R^{2}}{1+R}+\frac{16}{15}\right)
\end{aligned}
$$

where $R \equiv 3 \rho_{\mathrm{b}} / 4 \rho_{\gamma}, \rho_{\mathrm{b}}$ is the baryon energy density.

Replacing $\cos ^{2}\left(k r_{\mathrm{s}}\right)$ with its average value over an oscillation of $1 / 2$, we get the energy release per unit redshift

$$
\begin{aligned}
\frac{\mathrm{d} Q / \mathrm{d} z}{\rho_{\gamma}} & =\frac{-1}{3} \int \frac{\mathrm{d}^{3} k}{(2 \pi)^{3}} P_{\gamma}^{\mathrm{i}}(k) \frac{\mathrm{d} \Delta_{\gamma}^{2}}{\mathrm{~d} z} \\
& =3 \int \frac{\mathrm{d}^{3} k}{(2 \pi)^{3}} P_{\gamma}^{\mathrm{i}}(k) k^{2} \mathrm{e}^{-2 k^{2} / k_{\mathrm{D}}^{2}} \frac{\mathrm{d}\left(1 / k_{\mathrm{D}}^{2}\right)}{\mathrm{d} z} \\
& =\frac{4.3 A_{\zeta}}{k_{0}^{n_{\mathrm{s}}-1}} \frac{\mathrm{d}\left(1 / k_{\mathrm{D}}^{2}\right)}{\mathrm{d} z} \int \mathrm{d} k k^{n_{\mathrm{s}}} \mathrm{e}^{-2 k^{2} / k_{\mathrm{D}}^{2}} \\
& =\frac{4.3 A_{\zeta}}{k_{0}^{n_{\mathrm{s}}-1}} \frac{\mathrm{d}\left(1 / k_{\mathrm{D}}^{2}\right)}{\mathrm{d} z} 2^{-\left(3+n_{\mathrm{s}}\right) / 2} k_{\mathrm{D}}^{n_{\mathrm{s}}+1} \Gamma\left(\frac{n+1}{2}\right) .
\end{aligned}
$$

For a running index, the above integration must be done numerically. We are interested in radiation-dominated epoch where $\mu$ distortions are generated, and we have $H(z)=H_{0} \Omega_{r}^{1 / 2}(1+z)^{2}$ and $n_{\mathrm{e}}(z)=\left(n_{H 0}+2 n_{H \mathrm{e} 0}\right)(1+z)^{3} \equiv n_{\mathrm{e} 0}(1+z)^{3}$. In addition we 
have $k_{\mathrm{D}}=A_{\mathrm{D}}^{-1 / 2}(1+z)^{3 / 2}$ and $\mathrm{d}\left(1 / k_{\mathrm{D}}^{2}\right) / \mathrm{d} z=-3 A_{\mathrm{D}}(1+z)^{-4}$, and the above equation simplifies to

$\frac{\mathrm{d} Q / \mathrm{d} z}{\rho_{\gamma}}=-\frac{13 A_{\zeta}}{k_{0}^{n_{\mathrm{s}}-1}} 2^{-\left(3+n_{\mathrm{s}}\right) / 2} \Gamma\left(\frac{n_{\mathrm{s}}+1}{2}\right) A_{\mathrm{D}}^{\left(1-n_{\mathrm{s}}\right) / 2}(1+z)^{\left(3 n_{\mathrm{s}}-5\right) / 2}$

where

$A_{\mathrm{D}}=\frac{8 c}{135 H_{0} \Omega_{r}^{1 / 2} n_{\mathrm{e} 0} \sigma_{\mathrm{T}}}=5.92 \times 10^{10} \mathrm{Mpc}^{2}$.

The redshift and $n_{\mathrm{s}}$ dependence obtained above matches that of $\mathrm{Hu}$ et al. (1994). The diffusion scale $k_{\mathrm{D}}=46 \mathrm{Mpc}^{-1}$ at $z=5 \times 10^{4}$ and $k_{\mathrm{D}}=10^{4} \mathrm{Mpc}^{-1}$ at $z=2 \times 10^{6}$. Thus we are probing the primordial fluctuations on very small scales that are not accessible in any other way.

Thus for a Harrison-Zeldovich spectrum with $n_{\mathrm{s}}=1$

$$
\begin{gathered}
\frac{\mathrm{d} Q / \mathrm{d} z}{\rho_{\gamma}}=\frac{-7.8 \times 10^{-9}}{1+z} \\
\frac{Q_{z=2 \times 10^{6}}^{z=5 \times 10^{4}}}{\rho_{\gamma}}=2.9 \times 10^{-8} .
\end{gathered}
$$

We note the surprising fact that this redshift dependence is exactly the same as for the energy losses due to adiabatic cooling Eq. (13), as already pointed out by Chluba \& Sunyaev (2012). For the currently preferred value of $n_{\mathrm{s}}=0.96$ we have

$$
\begin{gathered}
\frac{\mathrm{d} Q / \mathrm{d} z}{\rho_{\gamma}}=\frac{-1 \times 10^{-8}}{(1+z)^{1.06}} \\
\frac{Q_{z=2 \times 10^{6}}^{z=5 \times 10^{4}}}{\rho_{\gamma}}=1.8 \times 10^{-8} .
\end{gathered}
$$

Figure 7 compares the rate of energy release multiplied by the blackbody visibility function, $G(z)(1+z) \mathrm{d} Q / \mathrm{d} z / E_{\gamma}$ for different initial power spectra with the energy losses due to the adiabatic cooling of baryons, $(1+z) S_{\text {Compton }} / E_{\gamma}$ obtained by solving Eq. (9) for the standard recombination history (Seager et al. 2000) calculated using the effective multilevel approach and taking recent corrections into account (Ali-Haïmoud \& Hirata 2011; Chluba \& Thomas 2011). The spike due to increase in Silk damping during recombination is clearly noticeable ${ }^{7}$. The smaller spikes due to HeII and HeI are also noticeable. Recombination of each species leads to a decrease in the number of particles, hence to adiabatic losses. But the photon diffusion length, and the associated energy release, increases owing to a decrease in the electron fraction. The transition from radiation to matter domination results in unnoticeable change in the slope of the curves. Even after recombination photons continue to mix on horizon scales because of free streaming, while the energy losses from Comptonization drop sharply as a result of depletion of electrons. We switch to the free-streaming solution described in Appendix A when diffusion length $2 \pi / k_{\mathrm{D}}$ becomes equal to the comoving particle horizon. In the blackbody photosphere region, the blackbody visibility function $G(z)$ makes the curves drop sharply. Global energy release and $y$-type distortions during the free streaming epoch after recombination are due to the superposition of blackbody spectra on horizon scales. The energy injected into $y$-type distortion during recombination in the peak $(800<z<1500)$ is $\Delta E / E \sim 10^{-8}$ for $n_{\mathrm{s}}=0.96$ (see

\footnotetext{
7 The actual energy injected in the recombination peak would be much less because of the transition to free streaming.
}

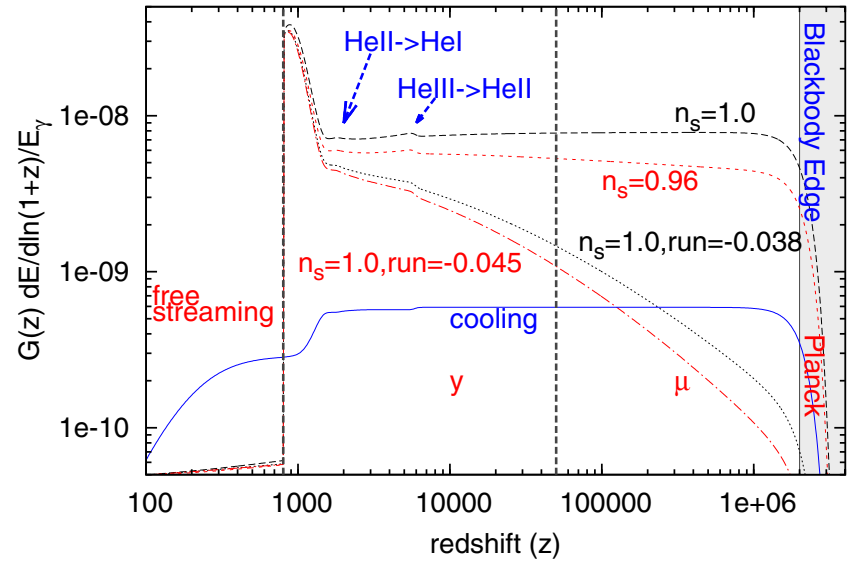

Fig. 7. Sketch of fractional rate of energy release due to Silk damping and free streaming for different initial power spectra. Also shown for comparison is the rate of energy loss due to adiabatic cooling of baryonic matter.

Table 1. Energy injection in $\mu$ distortions during $5 \times 10^{4}<z<2 \times$ $10^{6}$ for different initial power spectra without running compared with energy losses due to Bose-Einstein condensation.

\begin{tabular}{lc}
\hline \hline$n_{\mathrm{s}}$ & $\Delta E / E$ \\
\hline 1.07 & $6.8 \times 10^{-8}$ \\
1.04 & $4.7 \times 10^{-8}$ \\
1.0 & $2.9 \times 10^{-8}$ \\
0.96 & $1.8 \times 10^{-8}$ \\
0.92 & $1.1 \times 10^{-8}$ \\
\hline BEC & $-2.2 \times 10^{-9}$ \\
\hline
\end{tabular}

Table 2. Energy injection in $\mu$ distortions during $5 \times 10^{4}<z<2 \times 10^{6}$ for different initial power spectra with running compared with energy losses due to Bose-Einstein condensation.

\begin{tabular}{lcc}
\hline \hline$n_{\mathrm{s}}$ & $\mathrm{d} n_{\mathrm{s}} / \mathrm{d} \ln k$ & $\Delta E / E$ \\
\hline 1.07 & -0.05 & $2.2 \times 10^{-9}$ \\
1.07 & -0.035 & $5.7 \times 10^{-9}$ \\
1.07 & -0.02 & $1.6 \times 10^{-8}$ \\
1.04 & -0.05 & $1.6 \times 10^{-9}$ \\
1.04 & -0.035 & $4.1 \times 10^{-9}$ \\
1.04 & -0.02 & $1.1 \times 10^{-8}$ \\
1.0 & -0.05 & $1.1 \times 10^{-9}$ \\
1.0 & -0.038 & $2.2 \times 10^{-9}$ \\
1.0 & -0.035 & $2.6 \times 10^{-9}$ \\
1.0 & -0.02 & $6.9 \times 10^{-9}$ \\
\hline $\mathrm{BEC}$ & - & $-2.2 \times 10^{-9}$ \\
\hline
\end{tabular}

Notes. Energy injection values $\leq$ BEC value in magnitude are shown in red (in the electronic edition).

also footnote 7). The $y$ distortion due to mixing of blackbodies during free streaming after recombination and up to reionization, $20<z<800$, calculated using the result in the Appendix A, is $\Delta E / E \sim 10^{-10}$. These are, however, difficult to separate from much the larger thermal and non-linear Doppler $y$ distortion from reionization of $Y_{\mathrm{SZ}} \sim 10^{-7}$.

Energy releases for different power spectra, including the ones with running spectral index, are summarized in Tables 1 and 2 . The energy losses from adiabatic cooling of baryons during the same time period is $\sim 2.2 \times 10^{-9}$. This equals the energy release, for example, for a spectral index of $n_{\mathrm{s}}=1.0$, 
R. Khatri et al.: Does Bose-Einstein condensation of CMB photons cancel $\mu$ distortions...

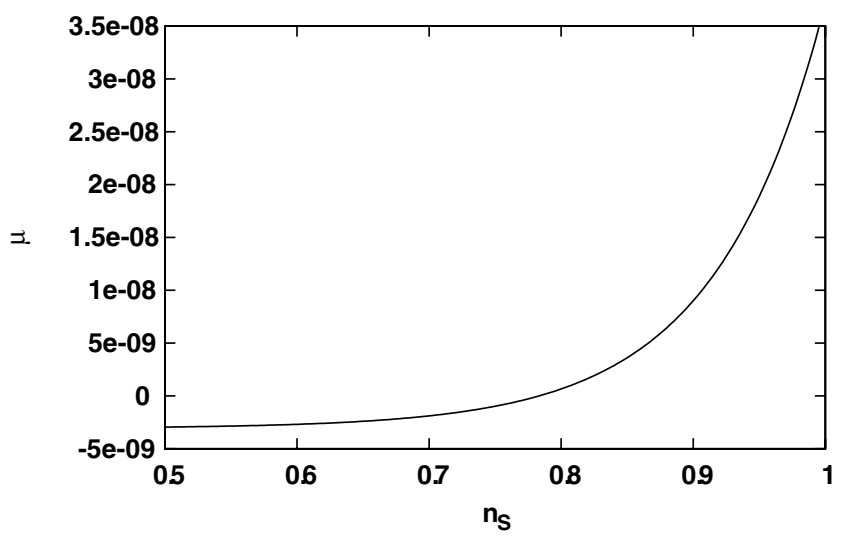

Fig. 8. $\mu=1.4 \Delta E / E$ as a function of spectral index $n_{\mathrm{s}}$ without running.

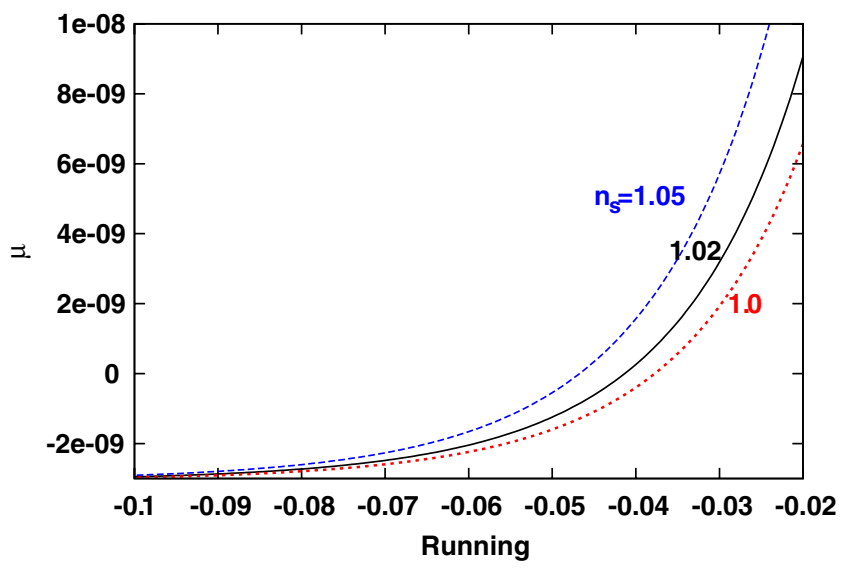

Fig. 9. $\mu=1.4 \Delta E / E$ as a function of running of the spectral index $r$ for three values of spectral index $n_{\mathrm{s}}$.

$\mathrm{d} n_{\mathrm{s}} / \mathrm{d} \ln k=-0.038$ for a running spectrum. The net $\mu$ distortion as a function of spectral index $n_{\mathrm{s}}$ without running is shown in Fig. 8 and for $n_{\mathrm{s}}=1.0,1.02,1.05$ as a function of the running of the index in Fig. 9. For low values of $n_{\mathrm{s}}$ and high negative values of running, Bose-Einstein condensation dominates, and net distortion approaches a constant value of $-3 \times 10^{-9}$. A detection or non-detection at a sensitivity of $10^{-9}$ of $\mu$-type distortion is thus a very sensitive probe of primordial power spectrum on small scales. Given the importance of the effective heating rate for the amplitude of the net distortion, it is important to carry out a more careful and refined calculation of the problem, which we present elsewhere (Chluba et al. 2012).

Over the years a number of ground- and balloon-based experiments that aimed at measuring the absolute CMB brightness have been conducted before COBE (Johnson \& Wilkinson 1987; Levin et al. 1992) and after it (Bersanelli et al. 1994; Fixsen et al. 2011, 2004; Raghunathan \& Subrahmnayan 2000; Staggs et al. 1996; Zannoni et al. 2008). The best limits on CMB $\mu$-type spectral distortions from these experiments are at the level of $10^{-4}-10^{-5}$ (Fixsen et al. 1996; Gervasi et al. 2008; Seiffert et al. 2011). Achieving a sensitivity of $10^{-9}$ would require subtracting foregrounds due to synchrotron emission, free free emission, dust emission, and spinning dust emission at the same precision level. The proposed experiment Pixie (Kogut et al. 2011) aims to achieve this goal by using 400 effective channels between frequencies of $30 \mathrm{GHz}$ to $6 \mathrm{THz}$. Their simulations indicate that an accuracy of $1 \mathrm{nK}$ in foreground subtraction is achievable. There is, however, some uncertainty in our understanding of the foregrounds and possible systematics as indicated by an observed excess signal at $3 \mathrm{GHz}$ by ARCADE (Fixsen et al. 2011) experiment, which is not completely explained by the current galactic and extragalactic emission models; nevertheless achievement of a goal of $10^{-9}$ in sensitivity, although challenging, seems possible in the near future.

\section{Conclusions}

Comptonization of CMB photons with slightly cooler electrons results in spectral distortions that then evolve (in the absence of any other physical process) towards the Bose-Einstein condensate solution, which is the equilibrium solution and consists of a blackbody spectrum with zero chemical potential, with the extra photons accumulating at zero frequencies (Illarionov \& Sunyaev 1975a; Landau \& Lifshitz 1980). This is because the radiation has more photons than can be accommodated in a Bose-Einstein spectrum with non-negative chemical potential at the electron temperature. However, in reality, bremsstrahlung and double Compton scattering destroy extra photons at low frequencies. Thus in practice we have the high-frequency spectrum evolving towards a blackbody with the extra photons slowly moving down in frequency to be eaten up by bremsstrahlung and double Compton scattering. This is what is seen in the numerical solution in Fig. 6.

The difficulty of conserving photon number is the reason Bose-Einstein condensation (or accumulation of photons at low frequencies) is not observed in astrophysical systems. BoseEinstein condensation of photons has only been achieved in the laboratory very recently by Klaers et al. (2010). In the real Universe we also have inevitable sources of energy injection into the $\mathrm{CMB}$, such as the $\mathrm{SZ}$ effect after reionization, which creates distortions of similar magnitude but with opposite signs canceling and overwhelming this effect. The situation is much more interesting for $\mu$ distortions, which can be created only at high redshifts and thus have no foregrounds at lower redshifts. In standard cosmology, positive $\mu$ distortions are created by dissipation of acoustic waves on small scales due to photon diffusion. For the currently allowed values of cosmological parameters, there is a possibility that the positive $\mu$ distortions from photon diffusion can almost exactly cancel the negative $\mu$ distortions from Bose-Einstein condensation of $\mathrm{CMB}$, leading to a net distortion that is much smaller. Bose-Einstein condensation can even dominate, leading to a net distortion $\mu \sim-3 \times 10^{-9}$ to which Silk damping contributes, but only as a small perturbation. Nevertheless, we must emphasize that the photon number conserving Comptonization, along with differences in adiabatic indices of radiation and matter, creates a unique system in the early Universe in which photons can begin to evolve towards a Bose-Einstein condensate. It is a remarkable coincidence that a completely unrelated physical process, diffusion damping of primordial perturbations, can produce distortions of almost exactly the same or greater magnitude, leading to the suppression of this unique effect in astrophysics. We thus arrive at an intriguing conclusion: even a null result, non-detection of $\mu$-type distortion at a sensitivity of $10^{-9}$, rather than just placing a upper limit, actually gives a quantitative measure of the primordial small-scale power spectrum. The importance of improving the experimental sensitivity to reach this critical value cannot be overemphasized.

\section{Appendix A: Erasure of perturbations due to free streaming}

We can derive the solution for erasure if perturbations on horizon scales due to free streaming as follows. We start with the 
Boltzmann hierarchy, ignoring quadrupole and higher order moments. We define multipole $(\ell)$ moments of the temperature anisotropy $\Delta T / T \equiv \Theta=\sum_{\ell}(-i)^{\ell}(2 \ell+1) \mathcal{P}_{\ell}(\hat{k} . \hat{n}) \Theta_{\ell}$, where $\mathcal{P}_{\ell}$ is the Legendre polynomial, and $\hat{k} . \hat{n}$ the angle between photon momentum and comoving wavenumber. In the limit of zero Thomson optical depth we get

$$
\begin{array}{r}
\frac{\mathrm{d} \Theta_{0}}{\mathrm{~d} \eta}+k \Theta_{1}=0 \\
\frac{\mathrm{d} \Theta_{1}}{\mathrm{~d} \eta}-\frac{k}{3} \Theta_{0}=\frac{k \psi}{3},
\end{array}
$$

where $\eta=\mathrm{d} t / a$ is the conformal time. Using the fact that in the matter-dominated era the potential is suppresses by a factor of 9/10 compared to primordial value on large scales, we have $\Theta_{0}=-2 \psi / 3$ (see for example Dodelson 2003) and looking for a solution with time dependence of type $\Theta_{\ell} \propto \mathrm{e}^{\mathrm{i} \int \mathrm{d} \eta \omega}$ we get $\omega=\mathrm{i} k / \sqrt{6}$ Thus free streaming damps the perturbations by a factor of $\mathrm{e}^{-\int \mathrm{d} \eta k / \sqrt{6}}=\mathrm{e}^{-0.4 \int \mathrm{d} \eta k}$. Finally we want to mention that this solution is very approximate, and in principle we should also take higher $\ell$ modes into account. For example, on going up to $\ell=3$ we get for the damping factor $\mathrm{e}^{-0.3 \int \mathrm{d} \eta k}$. Also for free streaming we have super position of blackbodies, and the $y$ distortion is given by (Chluba \& Sunyaev 2004) $Y_{\mathrm{SZ}}=1 / 2\left\langle\Theta^{2}\right\rangle=1 / 32\left\langle\delta_{\gamma}^{2}\right\rangle$, and so the equivalent energy release is $\Delta E / E=4 Y_{\mathrm{SZ}}=1 / 8\left\langle\delta_{\gamma}^{2}\right\rangle$.

Acknowledgements. We would like to thank Matias Zaldarriaga for important remarks on the manuscript. We would also like to thank Yacine Ali-Haïmoud for careful reading and comments on the manuscript.

\section{References}

Ali-Haïmoud, Y., \& Hirata, C. M. 2011, Phys. Rev. D, 83, 043513 Arons, J. 1971, ApJ, 164, 437

Bersanelli, M., Bensadoun, M., de Amici, G., et al. 1994, ApJ, 424, 517

Burigana, C., Danese, L., \& de Zotti, G. 1991, A\&A, 246, 49

Chluba, J. 2005, Ph.D. Thesis, LMU München

Chluba, J., \& Sunyaev, R. A. 2004, A\&A, 424, 389

Chluba, J., \& Sunyaev, R. A. 2006, A\&A, 458, L29

Chluba, J., \& Thomas, R. M. 2011, MNRAS, 412, 748

Chluba, J., \& Sunyaev, R. A. 2012, MNRAS, 419, 1294

Chluba, J., Khatri, R., \& Sunyaev, R. A. 2012, MNRAS, submitted [arXiv: 1202.0057$]$

Danese, L., \& de Zotti, G. 1982, A\&A, 107, 39
Dodelson, S. 2003, Modern cosmology Scott Dodelson (Amsterdam: Netherlands Academic Press)

Dubrovich, V. K. 1975, Sov. Astron. Lett., 1, 196

Fixsen, D. J., Cheng, E. S., Gales, J. M., et al. 1996, ApJ, 473, 576

Fixsen, D. J., Kogut, A., Levin, S., et al. 2004, ApJ, 612, 86

Fixsen, D. J., Kogut, A., Levin, S., et al. 2011, ApJ, 734, 5

Gervasi, M., Zannoni, M., Tartari, A., Boella, G., \& Sironi, G. 2008, ApJ, 688, 24

Hu, W., \& Silk, J. 1993, Phys. Rev. D, 48, 485

Hu, W., Scott, D., \& Silk, J. 1994, ApJ, 430, L5

Illarionov, A. F., \& Syunyaev, R. A. 1972, Sov. Ast., 16, 45

Illarionov, A. F., \& Sunyaev, R. A. 1975a, Sov. Ast., 18, 413

Illarionov, A. F., \& Sunyaev, R. A. 1975b, Sov. Ast., 18, 691

Johnson, D. G., \& Wilkinson, D. T. 1987, ApJ, 313, L1

Kaiser, N. 1983, MNRAS, 202, 1169

Keisler, R., Reichardt, C. L., Aird, K. A., et al. 2011, ApJ, 743, 28

Klaers, J., Schmitt, J., Vewinger, F., \& Weitz, M. 2010, Nature, 468, 545

Kogut, A., Fixsen, D. J., Chuss, D. T., et al. 2011, JCAP, 07, 025

Komatsu, E., Smith, K. M., Dunkley, J., et al. 2011, ApJS, 192, 18

Kompaneets, A. S. 1956, Zh. Eksp. Teor. Fiz., 31, 876

Landau, L. D., \& Lifshitz, E. M. 1980, Statistical physics, Part. 1 (Oxford: Butterworth-Heinemann)

Lehners, J.-L., McFadden, P., Turok, N., \& Steinhardt, P. J. 2007, Phys. Rev. D, 76,103501

Levich, E. V., \& Sunyaev, R. A. 1971, Sov. Ast., 15, 363

Levin, S., Bensadoun, M., Bersanelli, M., et al. 1992, ApJ, 396, 3

Lifshitz, E. M., \& Pitaevskii, L. P. 1981, Physical Kinetics (Oxford: ButterworthHeinemann)

Ma, C.-P., \& Bertschinger, E. 1995, ApJ, 455, 7

Mukhanov, V. F., \& Chibisov, G. V. 1981, Sov. J. Exp. Theor. Phys. Lett., 33, 532

Peebles, P. J. E. 1968, ApJ, 153, 1

Raghunathan, A., \& Subrahmnayan, R. 2000, JApA, 20, 1

Rubiño-Martín, J. A., Chluba, J., \& Sunyaev, R. A. 2006, MNRAS, 371, 1939

Rubiño-Martín, J. A., Chluba, J., \& Sunyaev, R. A. 2008, A\&A, 485, 377

Seager, S., Sasselov, D. D., \& Scott, D. 2000, ApJS, 128, 407

Seiffert, M., Fixsen, D. J., Kogut, A., et al. 2011, ApJ, 734, 6

Silk, J. 1968, ApJ, 151, 459

Staggs, S. T., Jarosik, N. C., Wilkinson, D. T., \& Wollack, E. J. 1996, ApJ, 458, 407

Syunyaev, R. A. 1971, Sov. Ast., 15, 190

Sunyaev, R. A., \& Chluba, J. 2009, Astron. Nachr., 330, 657

Sunyaev, R. A., \& Zeldovich, Y. B. 1970a, Ap\&SS, 9, 368

Sunyaev, R. A., \& Zeldovich, Y. B. 1970b, Ap\&SS, 7, 20

Weinberg, S. 2008, Cosmology (Oxford: Oxford University Press)

Zannoni, M., Tartari, A., Gervasi, M., et al. 2008, ApJ, 688, 12

Zel'Dovich, Y. B., \& Levich, E. V. 1969, Sov. J. Exp. Theor. Phys., 28, 1287

Zel'Dovich, Y. B., \& Levich, E. V. 1970, Sov. J. Exp. Theor. Phys. Lett., 11, 35

Zeldovich, Y. B., \& Sunyaev, R. A. 1969, Ap\&SS, 4, 301

Zeldovich, Y. B., Kurt, V. G., \& Syunyaev, R. A. 1968, Zh. Eksp. Teor. Fiz., 55, 278

Zeldovich, Y. B., Illarionov, A. F., \& Syunyaev, R. A. 1972, Sov. J. Exp. Theor. Phys., 35, 643 